\title{
Intermittent fasting is neuroprotective in focal cerebral ischemia by minimizing autophagic flux disturbance and inhibiting apoptosis
}

\author{
JI HEUN JEONG ${ }^{1}$, KWANG SIK YU ${ }^{1}$, DONG HO BAK ${ }^{1}$, JE HUN LEE ${ }^{1}$, NAM SEOB LEE ${ }^{1}$, \\ YOUNG GIL JEONG ${ }^{1}$, DONG KWAN KIM ${ }^{2}$, JWA-JIN KIM ${ }^{3,4}$ and SEUNG-YUN HAN ${ }^{1}$
}

\author{
Departments of ${ }^{1}$ Anatomy and ${ }^{2}$ Physiology, College of Medicine, Konyang University, Seo-gu, Daejeon 302-718; \\ ${ }^{3}$ Department of Anatomy, Brain Research Institute, College of Medicine, Chungnam National University, \\ Jung-gu, Daejeon 301-747; ${ }^{4}$ LES Corporation Inc., Yuseong-gu, Daejeon 305-335, Republic of Korea
}

Received June 23, 2015; Accepted August 12, 2016

DOI: $10.3892 / \mathrm{etm} .2016 .3852$

\begin{abstract}
Previous studies have demonstrated that autophagy induced by caloric restriction (CR) is neuroprotective against cerebral ischemia. However, it has not been determined whether intermittent fasting (IF), a variation of CR, can exert autophagy-related neuroprotection against cerebral ischemia. Therefore, the neuroprotective effect of IF was evaluated over the course of two weeks in a rat model of focal cerebral ischemia, which was induced by middle cerebral artery occlusion and reperfusion (MCAO/R). Specifically, the role of autophagy modulation as a potential underlying mechanism for this phenomenon was investigated. It was demonstrated that IF reduced infarct volume and brain edema, improved neurobehavioral deficits, and rescued neuronal loss after $\mathrm{MCAO} / \mathrm{R}$. Furthermore, neuronal apoptosis was decreased by IF in the rat cortex. An increase in the number of autophagosomes (APs) was demonstrated in the cortices of IF-treated rats, using immunofluorescence staining and transmission electron microscopy. Using immunoblots, an IF-induced increase was detected in microtubule-associated protein 1 light chain 3 (LC3)-II, Rab7, and cathepsin D protein levels, which corroborated previous morphological studies. Notably, IF reduced the accumulation of APs and p62, demonstrating that IF attenuated the MCAO/R-induced disturbance of autophagic flux in neurons. The findings of the present study
\end{abstract}

Correspondence to: Professor Seung-Yun Han, Department of Anatomy, College of Medicine, Konyang University, 685 Gasuwon-dong, Seo-gu, Daejeon 302-718, Republic of Korea E-mail: jjzzy@konyang.ac.kr

Professor Jwa-Jin Kim, Department of Anatomy, Brain Research Institute, College of Medicine, Chungnam National University, 99 Daehak-ro, Jung-gu, Daejeon 301-747, Republic of Korea E-mail:kjj1021@konyang.ac.kr

Key words: intermittent fasting, autophagic flux, middle cerebral artery occlusion/reperfusion, apoptosis suggest that IF-induced neuroprotection in focal cerebral ischemia is due, at least in part, to the minimization of autophagic flux disturbance and inhibition of apoptosis.

\section{Introduction}

Neuronal death induced by cerebral ischemia is a major cause of high morbidity and mortality (1). Although previous studies have yielded encouraging results when investigating therapeutic candidates using animal models of cerebral ischemia $(2,3)$, no pharmacological strategies are currently effective in the clinical setting.

Autophagy is a pathway that is responsible for maintaining cellular homeostasis by destroying aggregated biomolecules (4). Upon starvation or hypoxia, autophagosomes (APs) are formed and degraded upon fusion with a lysosome (5). 'Autophagic flux' is defined as a well-balanced cycle that consists of the generation and degradation of APs. Notably, it has been previously reported that autophagic flux disturbance may contribute to ischemia-induced neuronal death (6). This finding has prompted the hypothesis that the promotion of autophagic flux may be a potent therapeutic strategy for cerebral ischemia.

A previous study demonstrated that caloric restriction (CR) alleviates neurodegeneration through the activation of autophagy (7). Intermittent fasting (IF), a variation of CR, is a defined regimen that consists of intervals between meals. In the past decade, IF has gained attention because of its beneficial role in weight loss (8). Previous studies have indicated that IF is neuroprotective due to its role in attenuating neuroinflammation (9) and increasing neurogenesis (10). However, to the best of our knowledge, there are no reports on the possible modulation of autophagy activity as well as apoptosis as a therapeutic mechanism underlying IF on cerebral ischemia.

Therefore, the present study aimed to test the hypothesis whether i) IF confers neuroprotection against cerebral ischemia via modulation of autophagy; and ii) autophagic modulation by IF is accompanied by an inhibition of apoptosis in a rat model of focal cerebral ischemia. 


\section{Materials and methods}

Animals. A total of 128 male Sprague-Dawley rats (weight, 220-250 g; age, 6 weeks) were purchased from a breeding colony maintained at the Korea Research Institute of Bioscience and Biotechnology (Daejeon, Korea) and maintained at a constant temperature $\left(23^{\circ} \mathrm{C}\right)$ and humidity (40-50\%) with a 12-h light/dark cycle. They were allowed free access to water and food. All experimental procedures were carried out in accordance with the National Institutes of Health (NIH, Bethesda, MD, USA). Rats were randomly assigned to four groups: Control rats $(n=32)$ were permitted free access to food without any subsequent surgery; IF, rats $(n=32)$ were subjected to two weeks of IF without any surgery; middle cerebral artery occlusion and reperfusion $(\mathrm{MCAO} / \mathrm{R})$, rats $(\mathrm{n}=32)$ were permitted free access to food and subsequent $\mathrm{MCAO} / \mathrm{R}$; and $\mathrm{IF}+\mathrm{MCAO} / \mathrm{R}$, rats $(\mathrm{n}=32)$ were subjected to IF with subsequent $\mathrm{MCAO} / \mathrm{R}$. To stimulate two weeks of IF, a diet schedule was designed consisting of seven cycles of $24 \mathrm{~h}$ fasting and $24 \mathrm{~h}$ feeding. During the IF period, all rats were allowed free access to water.

$M C A O / R$. Rats were subjected to $\mathrm{MCAO} / \mathrm{R}$ as described in previous studies $(11,12)$. Briefly, rats were anesthetized via intraperitoneal injection of $30 \mathrm{mg} / \mathrm{kg}$ zoletil (Virbac, Seoul, Korea). Following a midline neck incision, the left common carotid artery (CCA) and the external carotid artery were isolated and ligated. A clip was placed on the internal carotid artery (ICA) to prevent bleeding. After creating a small hole in the CCA, a 4-0 nylon monofilament with a silicon coated tip $(0.35-0.37 \mathrm{~mm})$ was inserted into the hole of the left CCA. Following removal of the clip at the ICA, the silicon-coated filament was placed into the ICA to occlude the origin of the middle cerebral artery (MCA) until resistance was felt. To produce reperfusion, the filament was removed after $2 \mathrm{~h}$ of occlusion, and the incision was sutured.

Measurement of infarct volume. Infarct volume was assessed using the 2,3,5-triphenyltetrazolium chloride (TTC) method (13). At $24 \mathrm{~h}$ post-reperfusion, the brains of rats ( $\mathrm{n}=5 /$ group) were cut into five slices (thickness, $2 \mathrm{~mm}$ ), and incubated in $2 \%$ TTC solution at $37^{\circ} \mathrm{C}$ in the dark for $30 \mathrm{~min}$. Brain slices were subsequently photographed. To calculate the percentage of brain infarct, the following formula was used: Infarct ratio $(\%)=($ total contralateral hemispheric volume - total ipsilateral hemispheric stained volume) / total contralateral hemispheric volume x 100 .

Assessment of brain edema. Brain edema was evaluated by assessing brain water content (14). At $24 \mathrm{~h}$ post-reperfusion, each ipsilateral hemisphere ( $\mathrm{n}=5 / \mathrm{group}$ ) was weighed to obtain the wet weight. Samples were dried at $70^{\circ} \mathrm{C}$ for $48 \mathrm{~h}$ and the dried weight was subsequently measured. Brain water content (\%) was calculated using the following formula: [(wet weight - dry weight) / wet weight] x 100.

Behavioral test. Two different behavioral tests were used in the present study. Initially, rats ( $\mathrm{n}=5 /$ group) at post-operative day (POD) 1 had their sensorimotor functions tested by modification of scoring scales consisting of 18 points, as reported by Garcia et al (15). Conversely, rats (n=5/group) at POD 1, 3, 5, and 7 had their motor coordination tested by rotarod test: Rats were placed on a rotarod drum and time $(\mathrm{sec})$ of latency to fall from the rod was measured thrice per day. Rotarod speed was fixed to $15 \mathrm{rpm}$ over a 5-min period. When rats fell off the rod, trials ended. Average latency was calculated for each testing day.

Neuronal counting. At $72 \mathrm{~h}$ post-reperfusion, rats ( $\mathrm{n}=3 /$ group) were perfused with $4 \%$ paraformaldehyde (PFA). Brains were removed, embedded in paraffin wax and serially sectioned at 5- $\mu \mathrm{m}$ thickness. Five sections were randomly selected according to anatomical landmarks corresponding to -1.4 to $-1.8 \mathrm{~mm}$ in the antero-posterior axis of the brain atlas and stained with $0.1 \%$ cresyl-violet solution. Mean number of morphologically intact neurons in the $1-\mathrm{mm}^{2}$ sample of the cortex were counted and averaged by three blinded observers using light microscopy. Only intact neurons with clear nuclei and large cell bodies were counted.

Neuronal apoptosis assay. In order to assess neuronal apoptosis, terminal deoxynucleotidyl transferase dUTP nick end labeling (TUNEL) assay was used. Five cortical sections of each rat ( $\mathrm{n}=3 /$ group) at POD 1 were prepared, using the random selection protocols mentioned, and stained using a commercial kit (Promega Corporation, Madison, WI, USA) according to the manufacturer's protocol. The mean number of TUNEL-positive cells in the cortical samples were blindly counted and averaged.

Immunofluorescence. Randomly selected five sections from each rat ( $n=3$ /group) were deparaffinized, hydrated in distilled water, and exposed an antigen through antigen retrieval process using tissue retrieval solution. Endogenous peroxidase activity was blocked with $3 \% \mathrm{H}_{2} \mathrm{O}_{2}$ for $1 \mathrm{~h}$ and sections were rinsed in phosphate-buffered saline (PBS). Subsequently, each of the sections were incubated in a humid chamber for $24 \mathrm{~h}$ at $4^{\circ} \mathrm{C}$ with primary rabbit polyclonal anti-light chain 3 (LC3) (PM036; MBL, Co., Ltd., Nagoya, Japan) and mouse monoclonal anti-NeuN (GTX30773; Gene Tex, Inc., Irvine, CA, USA) antibodies that were diluted in blocking solution to 1:200. Following three washes in PBS, the sections were incubated with secondary antibodies containing polyclonal goat anti-rabbit IgG (111-225-144) and anti-mouse IgG (115-165-003; both Jackson Immunoresearch, West Grove, PA, USA) at a dilution of 1:200 for $4 \mathrm{~h}$ at room temperature, respectively. The sections were subsequently washed with PBS and mounted with a coverslip. Images were captured by a laser confocal microscope (LSM-700; Carl Zeiss AG, Oberkochen, Germany).

Transmission electron microscopy. Rats ( $\mathrm{n}=3 /$ group) at $12 \mathrm{~h}$ post-reperfusion were perfused with $0.1 \mathrm{M}$ phosphate buffer (pH 7.4) containing 2\% PFA and 2\% glutaraldehyde. Brains were removed, post-fixed for $24 \mathrm{~h}$, immersed in $1 \%$ osmium tetroxide in $0.1 \mathrm{M}$ cacodylate buffer and subsequently embedded in an epoxy resin. Ultrathin sections $(50 \mathrm{~nm})$ of the cortical regions were obtained, stained with $3 \%$ lead citrate, and examined using a transmission electron microscope (TEM; HT7700; Hitachi, Ltd., Tokyo, Japan). 
Western blot analysis. At $24 \mathrm{~h}$ post-reperfusion, the cortical tissues of each rat ( $n=5 /$ group) in the left MCA territory were rapidly dissected. Tissue samples were homogenized by adding lysis buffer and a protease inhibitor cocktail prior to centrifugation at $13,800 \times \mathrm{g}$ for $10 \mathrm{~min}$ at $4^{\circ} \mathrm{C}$. The total protein concentration of the supernatant was determined using a BCA protein assay (Pierce Biotechnology, Inc., Rockford, IL, USA). Subsequently, the protein sample was separated by $10 \%$ SDS-PAGE and transferred onto a polyvinylidenedifluoride membrane (Bio-Rad Laboratories, Inc., Hercules, CA, USA), which was blocked with 5\% nonfat milk in Tris-buffered saline with $0.1 \%$ Tween 20 (TBS-T) for $2 \mathrm{~h}$ at room temperature. The membranes were incubated with the following primary antibodies (diluted to 1:1,000) overnight at $4^{\circ} \mathrm{C}$ : Mouse monoclonal anti- $\beta$-actin (sc-47778), rabbit polyclonal anti-LC3 (sc-28266), rabbit polyclonal anti-beclin1 (sc-11427), mouse monoclonal anti-p62 (1:1,000; sc-28359), rabbit polyclonal anti-Rab7 (sc-10767) and goat polyclonal anti-cathepsin D (sc-6486). All primary antibodies were purchased from Santa Cruz Biotechnology, Inc., (Dallas, TX, USA). The membranes were washed three times for $10 \mathrm{~min}$ in TBS-T and further incubated for $2 \mathrm{~h}$ at room temperature with horseradish peroxidase (HRP)-conjugated rabbit anti-goat IgG (P0449), rabbit anti-mouse IgG (P0260) and swine anti-rabbit IgG (P0217) secondary polyclonal antibodies (all 1:2,000; all Dako, Glostrup, Denmark). Following three washes in TBS-T, the membranes were observed using the enhanced chemiluminescent HRP substrate (WBKLS0500; EMD Millipore, Billerica, MA, USA). Densitometric analysis was performed using ImageJ software (Version 1.48; National Institutes of Health, Bethesda, MA, USA).

Statistical analysis. Data are presented as the mean \pm standard error of the mean. Comparisons of the data from the different groups were analyzed using one-way analysis of variance (PASW Statistics version 18; SPSS, Inc., Chicago, IL, USA). $\mathrm{P}<0.05$ was considered to indicate a statistically significant difference.

\section{Results}

IF diminishes MCAO/R-induced infarct volume, brain edema, and behavioral deficits. Initially, successful establishment of IF in rats was confirmed by evaluating body weight. Similar to a previous study (10), the body weights of IF rats were significantly reduced compared to the control rats $(170 \pm 20.29$ vs. $232 \pm 37.5 \mathrm{~g}$; $\mathrm{P}<0.001$; Fig. 1A). To evaluate the effects of IF on infarct volume, brain edema and behavioral deficits after MCAO/R, TTC staining was performed, brain water content was measured and behavioral tests were performed, respectively. There were no visibly infarcted areas in the control group, whereas the $\mathrm{MCAO} / \mathrm{R}$ group exhibited marked areas of infarction (Fig. 1B). The ipsilateral infarct ratio of the IF $+\mathrm{MCAO} / \mathrm{R}$ group was $\sim 1 / 3$ of that of the $\mathrm{MCAO} / \mathrm{R}$ group (19.59 \pm 1.40 vs. $58.23 \pm 1.71 \%$; $\mathrm{P}<0.001$; Fig. 1C). Furthermore, the degree of brain edema in the IF $+\mathrm{MCAO} / \mathrm{R}$ group was significantly decreased compared with that of the $\mathrm{MCAO} / \mathrm{R}$ group $(79.4 \pm 0.5$ vs. $81.1 \pm 0.7 \%$; $\mathrm{P}<0.01$; Fig. 1D). Two different behavioral tests were conducted to assess the role of IF in MCAO/R-associated sensorimotor deficits and motor incoordination. The MCAO/R group was significantly impaired in sensorimotor functions compared with the control group (11.5 \pm 1.9 vs. $17.8 \pm 0.5$; $\mathrm{P}<0.001$; Fig. 1E). However, the IF $+\mathrm{MCAO} / \mathrm{R}$ group exhibited significantly improved sensorimotor function compared with the $\mathrm{MCAO} / \mathrm{R}$ group $(14.1 \pm 1.6$ vs. $11.5 \pm 1.9 ; \mathrm{P}<0.01)$. In the rotarod task performed on POD $1,3,5$, and 7 , latencies to fall were significantly reduced in the $\mathrm{MCAO} / \mathrm{R}$ group compared with the control and IF groups at all time points except POD $1 \quad(\mathrm{P}<0.05$ and $\mathrm{P}<0.001$, respectively; Fig. $1 \mathrm{~F})$. As predicted, rats in the $\mathrm{IF}+\mathrm{MCAO} / \mathrm{R}$ group remained on the rotarod longer than those in the MCAO/R group at POD 3, 5, and 7. The average latency of the IF + MCAO/R group was significantly greater than the average latency of the $\mathrm{MCAO} / \mathrm{R}$ group at POD 7 (168.8 47 vs. $79.9 \pm 23.2 \mathrm{sec} ; \mathrm{P}<0.001)$. These findings demonstrated that IF decreased the infarct volume, reduced the degree of brain edema, and improved neurobehavioral deficits in rat models of cerebral ischemia.

IF attenuates neuronal death and apoptosis induced by $M C A O / R$. Cresyl-violet staining was used to assess the extent of neuronal death in the cortex at $72 \mathrm{~h}$ post-reperfusion. Although only minimal intact neurons were detected (black arrows; Fig. 2A), dying or dead neurons (black arrowheads) were markedly increased in the cortices of the $\mathrm{MCAO} / \mathrm{R}$ group. However, the number of intact neurons was largely preserved in the IF + MCAO/R group compared with the $\mathrm{MCAO} / \mathrm{R}$ group. The number of intact neurons in the cortex of the IF + MCAO/R group was significantly increased by $\sim 20$ fold (83.5 \pm 10.4 vs. $4.5 \pm 1.5$; $\mathrm{P}<0.001$; Fig. $2 \mathrm{~B})$ compared with the $\mathrm{MCAO} / \mathrm{R}$ group. To assess neuronal apoptosis, the TUNEL assay was used. Neurons undergoing apoptosis (white arrowheads; Fig. 2A) in the IF + MCAO/R group were markedly decreased in the cortex compared with the MCAO/R group. Apoptotic cortical neurons in the $\mathrm{IF}+\mathrm{MCAO} / \mathrm{R}$ group were significantly decreased by $\sim 25 \%$ compared with those in the $\mathrm{MCAO} / \mathrm{R}$ group $(22 \pm 2.2$ vs. $81.7 \pm 4.8 ; \mathrm{P}<0.001$; Fig. 2C). This data suggests that the neuroprotection induced by IF involves, at least in part, an attenuation of neuronal apoptosis.

IF activates neuronal autophagy and attenuates the $M C A O / R$-induced accumulation of APS. It was hypothesized that IF may be neuroprotective in focal cerebral ischemia by modulating autophagy activity. While neuronal APs (white arrows; Fig. 3A), as indicated by LC3 puncta surrounding the NeuN-immunopositive neuronal nuclei, were rarely detected in the cortices of the control group, these were markedly increased by IF. Furthermore, as expected, APs were abundantly present in cortical neurons in the MCAO/R group compared with the control group. However, APs in the IF $+\mathrm{MCAO} / \mathrm{R}$ group were decreased compared with the $\mathrm{MCAO} / \mathrm{R}$ group. Subsequently, in order to visualize APs directly, the cortical neuronal ultrastructure was observed with the aid of TEM (Fig. 3B). APs (black arrows) were identified by the presence of digested or partially digested organelles confined within a double membrane. APs in cortical neurons were increased in the IF group compared with the control group. The results of immunofluorescence 
A

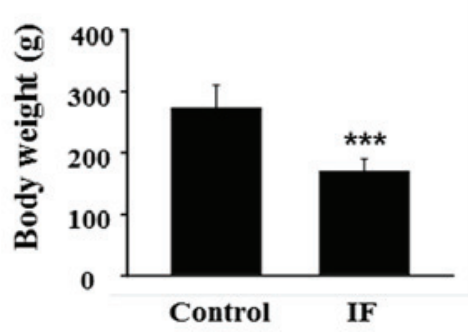

D

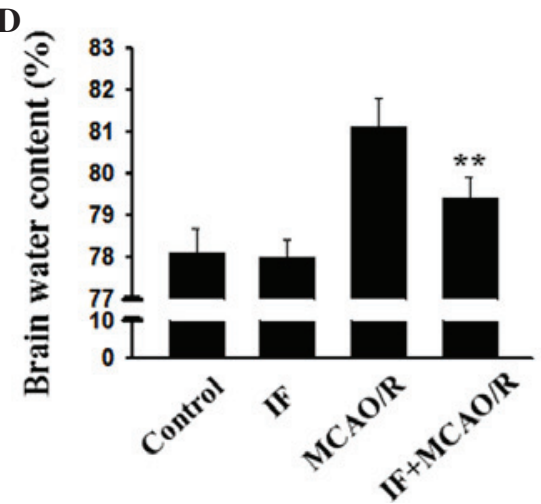

B

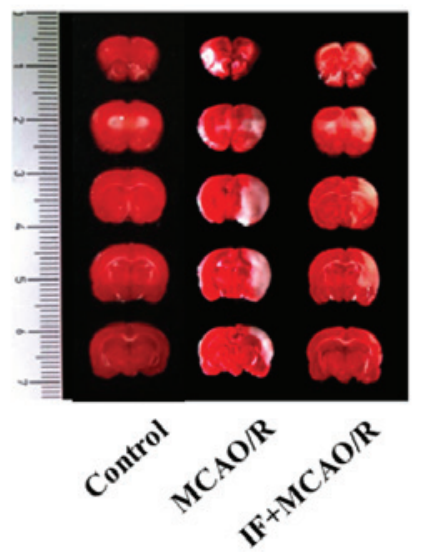

$\mathbf{E}$

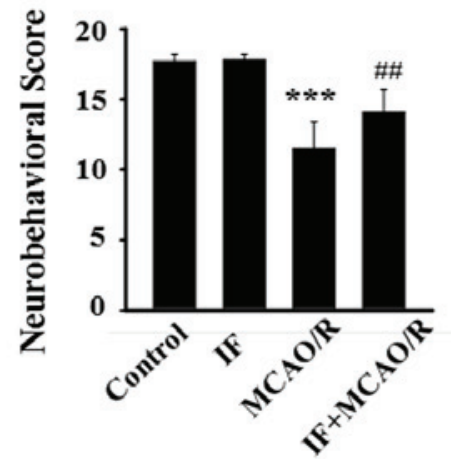

C

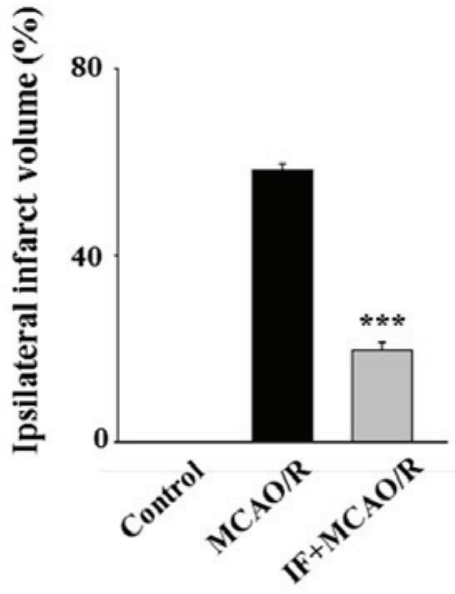

$\mathbf{F}$

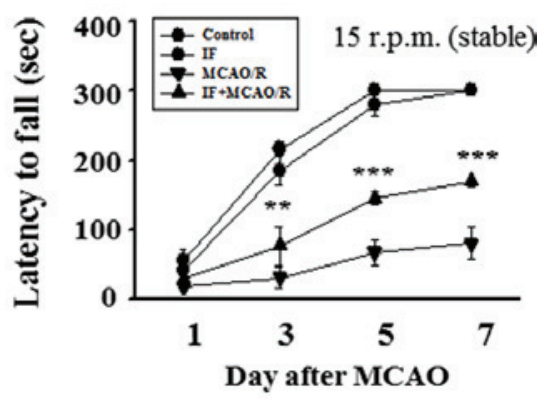

Figure 1. IF induces body weight loss, decreases infarct volume, reduces brain edema and improves neurobehavioral deficits after MCAO/R. (A) Body weight reduction induced by the IF regimen $\left({ }^{* * *} \mathrm{P}<0.001\right)$. (B) Representative images of tetrazolium chloride-stained brain sections $24 \mathrm{~h}$ after reperfusion. (C) Quantitative evaluation of ipsilateral infarct volumes in different groups ( $\mathrm{n}=5 / \mathrm{group} ;{ }^{* * * *} \mathrm{P}<0.001 \mathrm{vs}$. MCAO/R). (D) Percentage of brain water content in different groups ( $\mathrm{n}=5 /$ group; ${ }^{* *} \mathrm{P}<0.01 \mathrm{vs}$. MCAO/R). Effect of IF on (E) MCAO/R-induced neurological deficits, scaled by Garcia's method ( $=5 /$ group, ${ }^{* * * *} \mathrm{P}<0.001$ vs. control; ${ }^{\# / *} \mathrm{P}<0.01$ vs. $\left.\mathrm{MCAO} / \mathrm{R}\right)$ and $(\mathrm{F})$ on $\mathrm{MCAO} / \mathrm{R}$-induced motor deficits, which were estimated by the average latency to fall from a rotarod drum ( $\mathrm{n}=5 /$ group,${ }^{* *} \mathrm{P}<0.01$ and ${ }^{* * *} \mathrm{P}<0.001$ vs. MCAO/R). Data are presented as mean \pm standard error of the mean. IF, intermittent fasting; $\mathrm{MCAO} / \mathrm{R}$, middle cerebral artery occlusion and reperfusion.

staining and TEM also indicated that MCAO/R induced AP accumulation, whereas IF attenuated this. This data revealed that IF increased the basal activity of autophagy, and upon $\mathrm{MCAO} / \mathrm{R}, \mathrm{IF}$ attenuated the pathological accumulation of APs in cortical neurons.

IF attenuates MCAO/R-induced autophagic flux disturbance. The pathological accumulation of APs by MCAO/R may be triggered by two mechanisms: i) An increase in autophagy induction and ii) the disturbance of autophagic flux (16). To elucidate the mechanism, initially the amount of the conversion of LC3-I to its lipidated form was quantified, as LC3-II is a reliable marker for autophagy induction (17). In the present study, the ratio of LC3-II/LC3-I was increased in the IF, MCAO/R and $\mathrm{IF}+\mathrm{MCAO} / \mathrm{R}$ groups compared with the control group (Fig. 4A and B). As no difference was detected in LC3-II levels between the $\mathrm{MCAO} / \mathrm{R}$ and $\mathrm{IF}+\mathrm{MCAO} / \mathrm{R}$ groups, this suggests that neuronal autophagy was not significantly altered during autophagy induction in the IF $+\mathrm{MCAO} / \mathrm{R}$ group, compared with the $\mathrm{MCAO} / \mathrm{R}$ group. The effect of IF on autophagic flux in rat cortical neurons, with or without $\mathrm{MCAO} / \mathrm{R}$, was subsequently tested by measuring the levels of p62, cathepsin D, and Rab7 (18). Whereas p62, a cargo protein that undergoes autophagic degradation, is a negative-correlative marker for autophagic flux (19), cathepsin D and Rab7, an intralysosomal enzyme involved in the degradation of cellular waste and an essential protein for autophagosome-lysosome fusion, respectively, are both positive-correlative markers for autophagic flux $(20,21)$. Notably, the level of p62 was not altered in the IF group, but was significantly increased in the MCAO/R group compared with the control group ( $\mathrm{P}<0.01$; Fig. $4 \mathrm{C})$. This suggests that autophagic flux was maintained in the IF group, and disturbed in the $\mathrm{MCAO} / \mathrm{R}$ group. Additionally, the $\mathrm{IF}+\mathrm{MCAO} / \mathrm{R}$ group exhibited a significant decrease in neuronal p62 accumulation compared with the $\mathrm{MCAO} / \mathrm{R}$ group $(\mathrm{P}<0.01$; Fig. 4A and C). Furthermore, cathepsin D and Rab7 levels were increased in the IF + MCAO/R group compared with the MCAO/R group (Fig. 4A, D and E). These results suggest that IF attenuates MCAO/R-induced autophagic flux disturbance by promoting autophagic clearance, rather than by activating autophagy induction.

\section{Discussion}

In the present study, the involvement of autophagy in a rat model of $\mathrm{MCAO} / \mathrm{R}$ after IF was investigated. The findings 


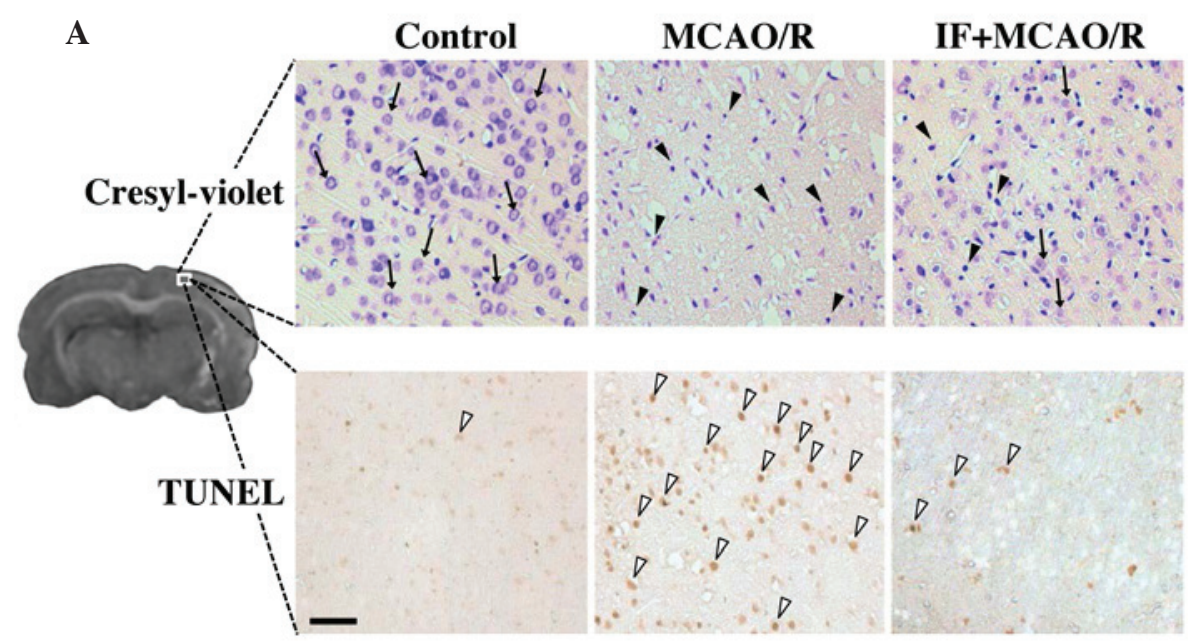

B

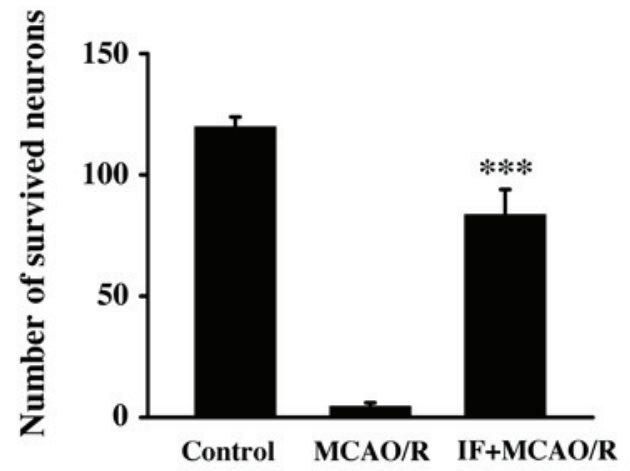

C

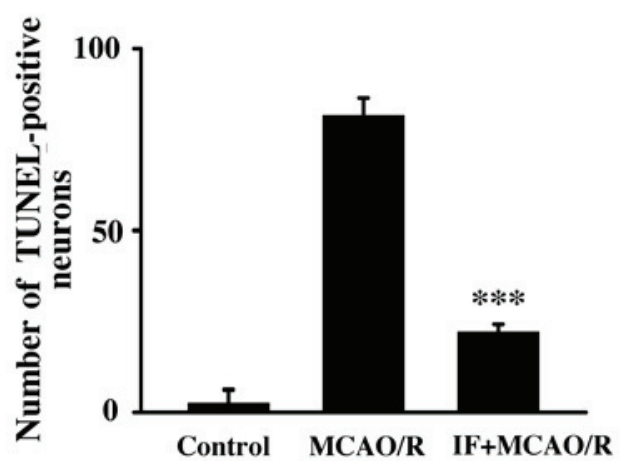

Figure 2. IF rescues cortical neurons from MCAO/R-induced neuronal death via the attenuation of apoptosis. (A) Representative images of cresyl violet- and TUNEL-stained cortical regions in different groups at $72 \mathrm{~h}$ post-reperfusion (scale bar, $50 \mu \mathrm{m}$ ). Intact neurons are indicated by black arrows, dead or dying neurons with pyknotic nuclei are indicated by black arrowheads, and apoptotic neurons with TUNEL-positive nuclei are indicated by white arrowheads. (B) Quantitative analysis of intact cortical neurons ( $\left.\mathrm{n}=3 / \mathrm{group} ;{ }^{* * * *} \mathrm{P}<0.001 \mathrm{vs} . \mathrm{MCAO} / \mathrm{R}\right)$. (C) Quantification of the number of TUNEL-positive neurons $\left(\mathrm{n}=3 /\right.$ group $;{ }^{* * *} \mathrm{P}<0.001$ vs. MCAO/R). Data are presented as mean \pm standard error of the mean. IF, intermittent fasting; MCAO/R, middle cerebral artery occlusion and reperfusion; hippo, hippocampus; TUNEL, terminal deoxynucleotidyl transferase dUTP nick-end labeling.

demonstrated that IF decreased MCAO/R-induced damages, including an increased infarct volume, brain edema, neurobehavioral deficits, and apoptotic neuronal death. In addition, IF was demonstrated to elevate basal autophagic activity and attenuate MCAO/R-induced autophagic flux disturbance using various methods. These results provide a potential direct mode of action of the neuroprotective role of IF, rather than indirect effects (hormonal or emotional influences). Therefore, preventing the deterioration of autophagic clearance, thereby minimizing autophagic flux disturbance, may be one of the direct effects of IF-induced neuroprotection.

Programmed cell death (PCD), which includes apoptosis (PCD type I) and autophagy (PCD type II), has an important role in cerebral ischemia (22). Whereas PCD type I always contributes to cell death, autophagy can lead to either cell survival or cell death, depending on the duration and severity of the process (23). The beneficial roles of autophagy in cellular survival are due to the elimination of damaged organelles and protein aggregates, as well as the facilitation of bioenergetic homeostasis (24). Previous studies have demonstrated that autophagy triggered by nutrient and growth factor deprivation has a pro-survival function at the cellular and organismal levels during instances of endoplasmic reticulum stress, development, microbial infection, and diseases characterized by the accumulation of protein aggregates $(25,26)$. In contrast, it has been demonstrated that autophagy can also promote cell death (27). When cells receive continuous or extremely stressful stimuli, PCD type II may occur (28). PCD type II has been implicated in various neurodegenerative diseases, including Alzheimer's disease and Parkinson's disease, as well as in acute injuries, such as stroke and trauma (29).

Although the key determinant of the paradoxical roles of autophagy in nervous system diseases remains to be addressed, it is generally accepted that 'autophagic stress', which is the sustained impairment of the balance between AP formation and degradation, contributes to PCD type II (30). If APs are efficiently degraded in autolysosomes, neurons can maintain homeostasis, leading to survival. However, when the late stages of the autophagic process (maturation or degradation) are defective, autophagic degradation is impaired, which can cause the pathologic accumulation of 
A

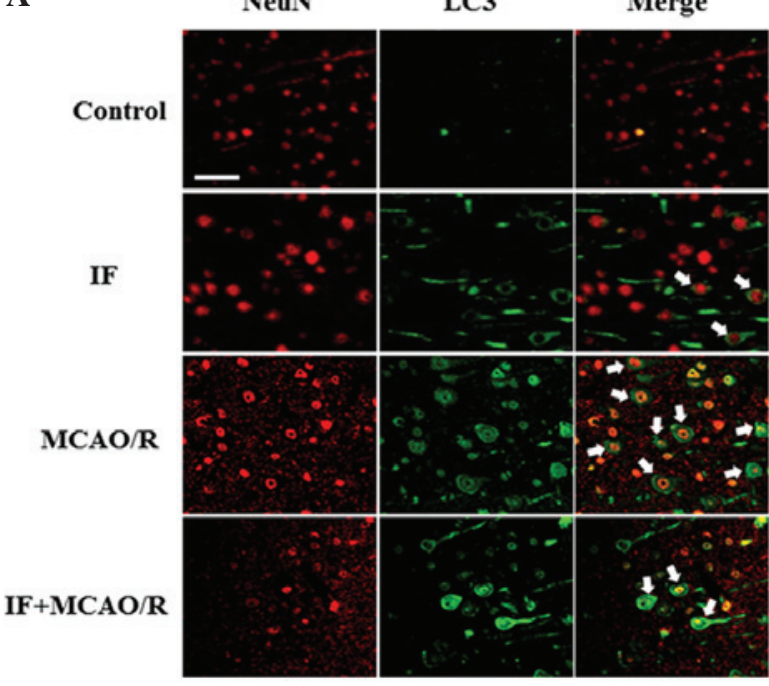

B

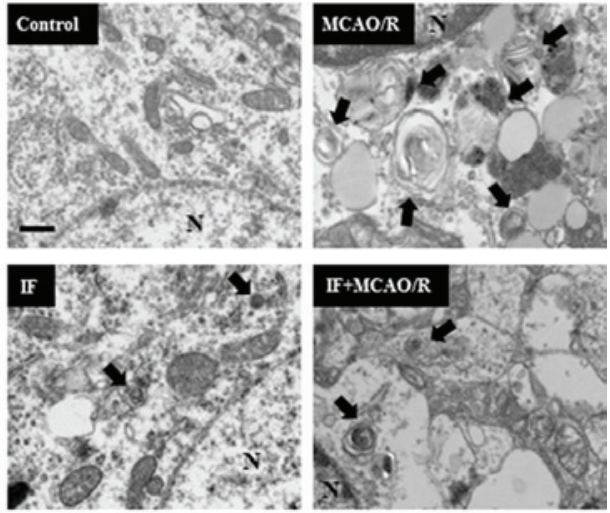

Figure 3. IF inhibits the accumulation of APs in cortical neurons after MCAO/R. (A) Representative images showing immunofluorescent staining for LC3 (green, AP) and NeuN (red, neuronal nuclei) in the cortex at $24 \mathrm{~h}$ post-reperfusion with or without IF preconditioning. Neurons with autophagosomes are indicated by arrows in merged images ( $\mathrm{n}=3 /$ group; scale bar, $25 \mu \mathrm{m}$ ). (B) Representative electron microphotographs showing APs in the cortical penumbra of $\mathrm{MCAO} / \mathrm{R}$ and IF $+\mathrm{MCAO} / \mathrm{R}$ groups at $12 \mathrm{~h}$ after reperfusion (n=3/group; scale bar, $200 \mathrm{~nm}$ ). Arrows indicate APs. IF, intermittent fasting; AP, autophagosomes; MCAO/R, middle cerebral artery occlusion and reperfusion; LC3, protein 1 lightchain 3.

A

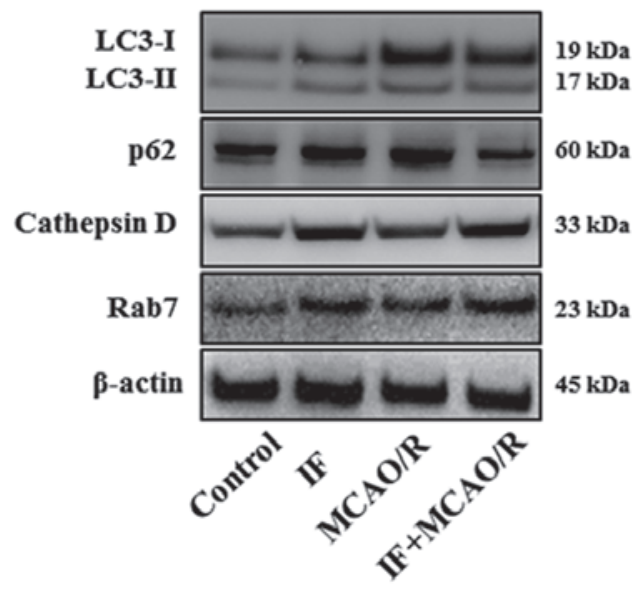

B

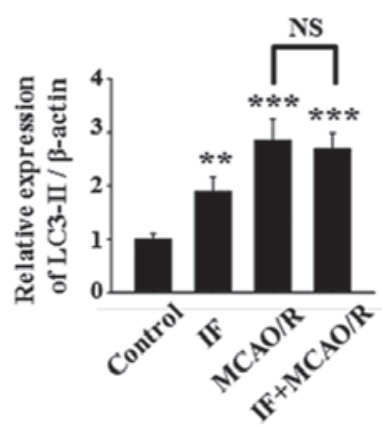

D

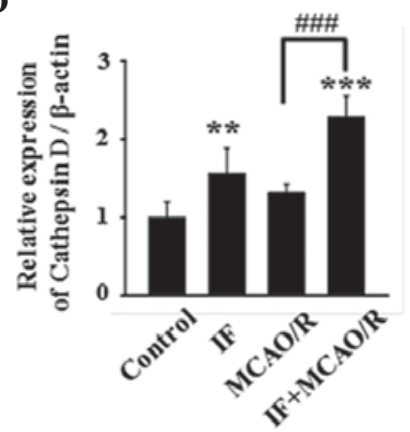

C

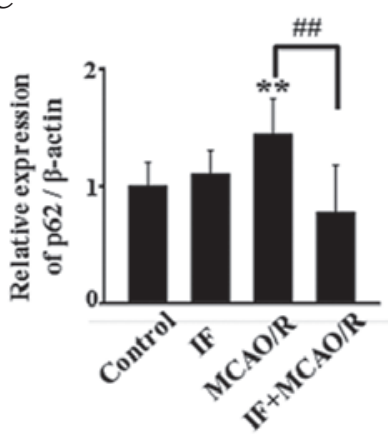

$\mathbf{E}$

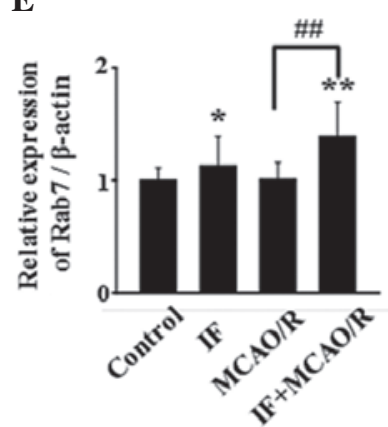

Figure 4. IF enhances the autophagic flux in cortical neurons after MCAO/R. (A) Representative image of immunoblots for LC3, p62, cathepsin D, and Rab7 in cortical homogenates in different groups at $12 \mathrm{~h}$ post-MCAO/R. (B) Quantification of LC3-II protein level. These data were quantified as a proportion of LC3-I intensity ( $\mathrm{n}=5$ /group; ${ }^{* *} \mathrm{P}<0.01$ and ${ }^{* * *} \mathrm{P}<0.001$ vs. control). (C-E) Quantification of p62, cathepsin D, and Rab7 protein levels, respectively. $\beta$-actin used as a loading control. ( $\mathrm{n}=5$ /group; ${ }^{*} \mathrm{P}<0.05,{ }^{* *} \mathrm{P}<0.01$ and ${ }^{* * *} \mathrm{P}<0.001$ vs. control; ${ }^{\# \#} \mathrm{P}<0.01$ and ${ }^{\# \# "} \mathrm{P}<0.001$ vs. MCAO/R). Data are presented as the mean \pm standard error of the mean. IF, intermittent fasting; MCAO/R, middle cerebral artery occlusion and reperfusion; LC3, protein light chain 3; NS, no statistical significance.

APs and subsequent neurodegeneration. In the present study, it was demonstrated that IF and MCAO/R increased APs in neurons, both morphologically and biochemically; whereas IF increased the degree of autophagic degradation, and $\mathrm{MCAO} / \mathrm{R}$ led to a decrease. Notably, IF prior to MCAO/R significantly increased the extent of autophagic degradation when compared with $\mathrm{MCAO} / \mathrm{R}$ alone. These observations support the hypothesis that IF preconditioning may confer an attenuation of MCAO/R-induced autophagic stress, and thus induce neuroprotection. 
If it is true that IF attenuates autophagic stress and thereby confers neuroprotection, the underlying mechanism of this phenomenon requires investigation. Furthermore, the possible relationship between autophagic flux restoration and apoptosis inhibition in neurons, both of which were observed as effects of IF on ischemic neurons, are yet to be elucidated. Recent studies have focused on the neuroprotective effects of ischemic preconditioning (IPC) to investigate these mechanisms. It has been reported that the autophagy inhibitor 3-methyladenine significantly suppressed IPC-induced neuroprotection and molecular chaperone upregulation in a rat model of cerebral ischemia (31). This data suggests that autophagy exerts IPC-induced neuroprotection via an upregulation of molecular chaperones. Furthermore, Carloni et al (32) revealed that the upregulation of autophagy was neuroprotective in a cerebral hypoxia-induced ischemia model in neonatal rats. According to their reports, an upstream autophagic activator, Beclin1, was markedly enhanced after hypoxia-ischemia and co-localized with TUNEL-positive cells, suggesting that the upregulation of autophagy and the promotion of apoptosis occurred simultaneously during ischemic injury. Moreover, inhibiting autophagy caused rapid cell death by apoptosis. Considering these previous reports, the findings of the present study suggest that IF increases basal activity of autophagy, thereby strengthening neuronal defense mechanisms, including molecular chaperone upregulation. Once toxic conditions are imposed on neurons, such as via $\mathrm{MCAO} / \mathrm{R}$, IF-induced autophagy activation confers neuroprotection through an inverse relationship with apoptosis.

In conclusion, the present study demonstrated that IF prevents neuronal damage in focal cerebral ischemia and that this protection is, at least partly, mediated by minimizing autophagic flux disturbance and the inhibition of apoptosis. Nevertheless, the possibility that IF induces neuroprotective effects via additional mechanisms such as systemic effects, (emotional and hormonal influences) cannot be excluded. Therefore, further studies with a more isolated approach may be necessary to examine the preventive role of IF in cerebral ischemia.

\section{Acknowledgements}

This study was equally supported by the Development of Forest Science and Technology (grant no. S111414L030100) and the Korea Research Foundation (grant no. NRF-2016R1C1B2012351).

\section{References}

1. Lipton P: Ischemic cell death in brain neurons. Physiol Rev 79: 1431-568, 1999.

2. Kim JH, Yu KS, Jeong JH, Lee NS, Lee JH, Jeong YG, Yoo YC and Han SY: All-trans-retinoic acid rescues neurons after global ischemia by attenuating neuroinflammatory reactions. Neurochem Res 38: 2604-2615, 2013.

3. Liu L, Zhang W, Wang L, Li Y, Tan B, Lu X, Deng Y, Zhang Y, Guo X, Mu J and Yu G: Curcumin prevents cerebral ischemia reperfusion injury via increase of mitochondrial biogenesis. Neurochem Res 39: 1322-1331, 2014.

4. Mizushima N: Autophagy: Process and function. Genes Dev 21: 2861-2873, 2007.

5. Kroemer G, Mariño G and Levine B: Autophagy and the integrated stress response. Mol Cell 40: 280-293, 2010.
6. Son JH, Shim JH, Kim KH, Ha JY and Han JY: Neuronal autophagy and neurodegenerative diseases. Exp Mol Med 44: 89-98, 2012.

7. Yang F, Chu X, Yin M, Liu X, Yuan H, Niu Y and Fu L: mTOR and autophagy in normal brain aging and caloric restriction ameliorating age-related cognition deficits. Behav Brain Res 264: 82-90, 2014.

8. Barnosky AR, Hoddy KK, Unterman TG and Varady KA: Intermittent fasting vs daily calorie restriction for type 2 diabetes prevention: A review of human findings. Transl Res 164: 302-311, 2014.

9. Fann DY, Santro T, Manzanero S, Widiapradja A, Cheng YL, Lee SY, Chunduri P, Jo DG, Stranahan AM, Mattson MP and Arumugam TV: Intermittent fasting attenuates inflammasome activity in ischemic stroke. Exp Neurol 257: 114-119, 2014.

10. Manzanero S, Erion JR, Santro T, Steyn FJ, Chen C, Arumugam TV and Stranahan AM: Intermittent fasting attenuates increases in neurogenesis after ischemia and reperfusion and improves recovery. J Cereb Blood Flow Metab 34: 897-905, 2014.

11. Calloni RL, Winkler BC, Ricci G, Poletto MG, Homero WM, Serafini EP and Corleta OC: Transient middle cerebral artery occlusion in rats as an experimental model of brain ischemia. Acta Cir Bras 25: 428-433, 2010.

12. Güzel A, Rölz R, Nikkhah G, Kahlert UD and Maciaczyk J: A microsurgical procedure for middle cerebral artery occlusion by intraluminal monofilament insertion technique in the rat: A special emphasis on the methodology. Exp Transl Stroke Med 6: 6-6, 2014.

13. Li F, Irie K, Anwer MS and Fisher M: Delayed Triphenyltetrazolium chloride staining remains useful for evaluating cerebral infarct volume in a rat stroke model. J Cereb Blood Flow Metab 17: 1132-1135, 1997.

14. Ito U, Ohno K, Nakamura R, Suganuma F and Inaba Y: Brain edema during ischemia and after restoration of blood flow. Measurement of water, sodium, potassium content and plasma protein permeability. Stroke 10: 542-547, 1979.

15. Garcia JH, Wagner S, Liu KF and Hu XJ: Neurological Deficit and Extent of neuronal necrosis attributable to middle cerebral artery occlusion in rats: Statistical validation. Stroke 26: 627-635, 1995.

16. Gu Z, Sun Y, Liu K, Wang F, Zhang T, Li Q, Shen L, Zhou L, Dong L, Shi N, et al: The role of autophagic and lysosomal pathways in ischemic brain injury. Neural Regen Res 8: 2117-2125, 2013.

17. Tanida I, Ueno T and Kominami E: LC3 and Autophagy. Methods Mol Biol 445: 77-88, 2008.

18. Su H, Li F, Ranek MJ, Wei N and Wang X: COP9 Signalosome regulates autophagosome maturation. Circulation 124: 2117-2128, 2011.

19. Bjørkøy G, Lamark T, Brech A, Outzen H, Perander M, Overvatn A, Stenmark H and Johansen T: p62/SQSTM1 forms protein aggregates degraded by autophagy and has a protective effect on huntingtin-induced cell death. J Cell Biol 171: 603-614, 2005.

20. Sarkar C, Zhao Z, Aungst S, Sabirzhanov B, Faden AI and Lipinski MM: Impaired autophagy flux is associated with neuronal cell death after traumatic brain injury. Autophagy 10: 2208-2222, 2014.

21. Hyttinen JM, Niittykoski M, Salminen A and Kaarniranta K: Maturation of autophagosomes and endosomes: A key role for Rab7. Biochim Biophys Acta 1833: 503-510, 2013.

22. Hossmann KA: Viability thresholds and the penumbra of focal ischemia. Ann Neurol 36: 557-565, 1994.

23. Klionsky DJ and Emr SD: Autophagy as a regulated pathway of cellular degradation. Science 290: 1717-1721, 2000.

24. Das G, Shravage BV and Baehrecke EH: Regulation and function of autophagy during cell survival and cell death. Cold Spring Harb Perspect Biol 4: pii: a008813, 2012.

25. Dalby KN, Tekedereli I, Lopez-Berestein G and Ozpolat B: Targeting the prodeath and prosurvival functions of autophagy as novel therapeutic strategies in cancer. Autophagy 6: 322-329, 2010.

26. Pankiv S, Clausen TH, Lamark T, Brech A, Bruun JA, Outzen H, Øvervatn A, Bjørkøy G and Johansen T: p62/SQSTM1 binds directly to Atg8/LC3 to facilitate degradation of ubiquitinated protein aggregates by autophagy. J Biol Chem 282: 24131-24145, 2007.

27. Mizushima N, Levine B, Cuervo AM and Klionsky DJ: Autophagy fights disease through cellular self-digestion. Nature 451: 1069-1075, 2008 
28. Bursch W, Ellinger A, Gerner $\mathrm{CH}$, Fröhwein U and Schulte-Hermann R: Programmed cell death (PCD). Apoptosis, autophagic PCD, or Others? Ann N Y Acad Sci 926: 1-12, 2000.

29. Yue Z, Friedman L, Komatsu M and Tanaka K: The cellular pathways of neuronal autophagy and their implication in neurodegenerative diseases. Biochim Biophys Acta 1793: 1496-1507, 2009.

30. Chu CT: Autophagic stress in neuronal injury and disease. J Neuropathol Exp Neurol 65: 423-432, 2006.
31. Meriin AB and Sherman MY: Role of molecular chaperones in neurodegenerative disorders. Int J Hyperthermia 21: 403-419, 2005.

32. Carloni S, Girelli S, Scopa C, Buonocore G, Longini M and Balduini W: Activation of autophagy and Akt/CREB signaling play an equivalent role in the neuroprotective effect of rapamycin in neonatal hypoxia-ischemia. Autophagy 6: 366-377, 2010. 